\title{
Research Paper \\ Relationship Between General Health and Demographic Characteristics of Family Caregivers of Stroke Survivors
}

\author{
Mahboobeh Tajvidi ${ }^{1}$, Asghar Dalvandi ${ }^{1}$, Robab Sahaf ${ }^{2}$, Mehdi Rahgozar ${ }^{3}$
}

1. Department of Nursing, University of Social Welfare and Rehabilitation Sciences, Tehran, Iran.

2. Iranian Research Center on Aging, University of Social Welfare and Rehabilitation Sciences, Tehran, Iran.

3. Department of Biostatistics, University of Social Welfare and Rehabilitation Sciences, Tehran, Iran.

Received: 26 Jul. 2017 Accepted: 15 Oct. 2017

Key words:

General health, stroke survivors, Family caregivers, Demographic characteristic
Citeation: Tajvidi M, Dalvandi A, Sahaf R, Rahgozar M. [Relationship Between General Health and Demographic Characteristics of Family Caregivers of Stroke Survivors (Persian)]. Iranian Journal of Ageing. 2018; 12(4):494-505. https://doi. org/10.21859/SIJA.12.4.494

https://doi.org/10.21859/SIJA.12.4.494

\begin{abstract}
A B STRACT
Objectives Caregiving of stroke survivors leads to several physiopsychlogical problems for family caregiver due to excessive demand by the stroke survivors. The aim of this study was to determine general health of the caregivers of stroke survivors and their relationship with demographic factors.

Methods \& Materials This was a cross-sectional study in which 60 caregivers of stroke survivors were selected by convenience sampling from Sina and Firouzgar Hospitals in Tehran province in Iran, in 2015. The data was collected by two questionnaires including demographic questionnaire and General Health Questionnaire (GHQ). The collected data were analyzed by descriptive and inferential statistics including mean and standard deviation using SPSS software (version 15).

Results The mean GHQ score was 61.41 \pm 14.9 ; in female caregiver, it was $63.07 \pm 16.09$, and in male caregiver, the score was $58.33 \pm 12.28$. Significant correlation found between overall GHQ score and either of the factors such as occupational status, age category, or type of caring $(P=0.01)$. Unemployment in caregiver, aging, and 24-hour caring of stroke survivors were related to a reduction in GHQ score. However, there was no significant association between $\mathrm{GHQ}$ of stroke caregivers and either of the factors such as $\operatorname{sex}(P=0.35)$, time of caring ( 0.24$)$, marital status $(P=0.71)$, or educational status.

Conclusion This study showed that family caregivers of stroke survivors experience deterioration of general health at different dimensions. Therefore, it is recommended that planning for general health and social support for caregivers should be done through policy making in health and treatment.
\end{abstract}

\section{Extended Abstract}

\section{Objectives}

aring for stroke survivors, due to their excessive demands, may create or exacerbate several physical and mental conditions for family caregivers. Therefore, the present study aims to determine the mean and standard deviation of the general health of family caregivers of stroke survivors and its subscales by men and women, as well as the relationship between the mean rate of general health or its subscales and demographic characteristics of family caregivers of a stroke survivor. Based on the

\section{* Corresponding Author:}

Asghar Dalvandi, PhD

Address: Department of Nursing, University of Social Welfare and Rehabilitation Sciences, Tehran, Iran.

Tel: +98 (21) 22180036

E-mail: as.dalvandi@uswr.ac.ir 
findings, the factors related to the general health of the caregivers of stroke survivors can be identified. Thus, the authorities of this area can take necessary steps to control the side effects of neglecting the general health of caregivers of stroke survivors, which can affect the different dimensions of their lives.

\section{Methods \& Materials}

A descriptive-analytical study was carried out by available sampling method on 60 family caregivers of stroke survivors referred to the outpatient clinics of Sina and Firoozgar Hospitals in 2015. Participants were the family caregivers aged at least 15 years and the stroke survivors aged over 50 years with 6 months after their stroke. The caregiver must not care any other patient than the stroke survivor. The data collection tool in this study was a two-part questionnaire. The first part was demographic data of caregivers, and the second part included a 28-item General Health Questionnaire (GHQ). The 28-item GHQ was first created by Goldberg in 1978 and consists of four 7-questions scales including physical scale, anxiety scale, social scale, and depression scale [1].

The validity of this questionnaire has been investigated and confirmed in previous studies. The reliability of this test in research conducted in Iran was 0.85 using alpha coefficient [4]. After getting the approval of the ethics committee of the research deputy of the University of Well-being and Rehabilitation Sciences in Tehran and presenting the official letter to the head of Sina and Firoozgar Hospitals in Tehran, the researcher went to the medical records department of the hospital to find the telephone numbers and addresses of the caregivers. Then, the researcher, in coordination with the caregivers, visited the caregivers in person or spoke over phone to them on various days and at different hours. After providing the necessary explanations, as- suring the confidentiality of their personal information, and obtaining written informed consent all the questionnaires were completed by the researcher through the interview. For statistical analysis, SPSS 15, as well as descriptive and inferential statistics including mean, standard deviation, Chi-square, and U-Mann-Whitney U-test were used. The significance level of all the tests was considered to be less than 0.05 .

\section{Results}

A total of 60 participants were included in this study. The mean age of the caregivers was $46.5 \pm 17.3$ years. Among them $43.3 \%$ were in the range of $31-59$ years, $33.3 \%$ had a high school diploma, $65 \%$ were women, $46.7 \%$ were spouses, $71.7 \%$ were married, and $75 \%$ were unemployed. In $90 \%$ of the caregivers, the duration of care for stroke survivors was greater than 6 months, and $51.7 \%$ of the patients needed around-theclock care.

The overall mean score of the general health of the caregivers was $61.41 \pm 14.9$, which was greater in women $(63.07 \pm 16.09)$ than in men $(33.58 \pm 12.28)$. It implies that women have problems with their general health. The mean score of general health subscales by gender is presented in Table 1 . The results of Mann-Whitney U-test and Chi-square test showed a significant relationship between total score of general health and job status, age group, or care status $(\mathrm{P}=0.01)$. Moreover, there is a significant relationship between physical health scale and age or care status $(\mathrm{P}=0.01)$. Significant relationship also exists between the social scale of health and job status $(\mathrm{P}=0.01)$, age group $(\mathrm{P}=0.33)$, educational status $(\mathrm{P}=0.01)$, or care status $(\mathrm{P}=0.01)$. The relationship between the depression scores and caregivers' care status also found to be significant $(\mathrm{P}=0.01)$.

Table 1. Mean \pm Standard Deviation of general health of family caregivers of stroke survivors by men and women

\begin{tabular}{|c|c|c|c|}
\hline \multirow{3}{*}{ General Health of Caregivers } & \multicolumn{3}{|c|}{ Frequency Distribution } \\
\hline & Men & Women & Total \\
\hline & Mean $\pm S D$ & Mean \pm SD & Mean \pm SD \\
\hline General health (total) & $33.58 \pm 28.12$ & $07.63 \pm 09.16$ & $41.61 \pm 9.14$ \\
\hline Physical subscale & $95.15 \pm 12.4$ & $25.17 \pm 3.5$ & $8.16 \pm 9.4$ \\
\hline Anxiety subscale & $9.16 \pm 5.4$ & $97.17 \pm 7.4$ & $6.17 \pm 6.4$ \\
\hline Social subscale & $05.13 \pm 7.3$ & $71.14 \pm 6.3$ & $15.14 \pm 7.3$ \\
\hline Depression subscale & $76.12 \pm 84.3$ & $56.13 \pm 5.13$ & $3.13 \pm 9.4$ \\
\hline
\end{tabular}




\section{Conclusion}

The findings of this study indicate that people who care for stroke survivors show a significant decline in their general health dimensions. Regarding the worsening of general health conditions in older caregivers, it is recommended to plan for the necessary social support from family caregivers by the policy makers in the field of health and treatment. The findings of this study also suggest the importance of the relationship between some demographic variables and the general health of family caregivers, which could provide information for developing strategies to improve the general health of family caregivers. Taking into account the cross-sectional nature, the location and sampling method of the study, and the lack of significant relationship with some of the demographic variables the generalization of the findings should be made cautiously. Therefore, studies with greater sample size in other areas are suggested, and the relationship between general health level and variables like gender and family relationship should be re-examined.

\section{Acknowledgments}

This research was extracted from the MSc. thesis of the first author in Department of Nursing, University of Social Welfare and Rehabilitation Sciences, and has been financially supported by the so-called University.

\section{Conflict of Interest}

The authors declared no conflicts of interest. 


\title{
ارتباط سلامت عمومى با مشخصات جمعيتشناختى مراقبان خانوادَّى بازماندكان سكته مغزى
}

\author{
محبوبه تجويدى'، "اصغر دالوندى'، رباب صحاف ؛ مهدى رهكذر”

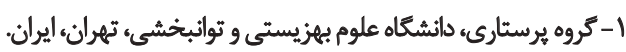

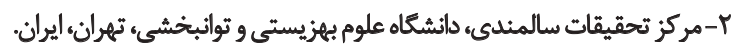

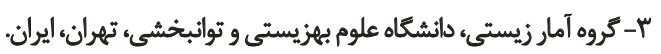

\begin{abstract}
حكبد

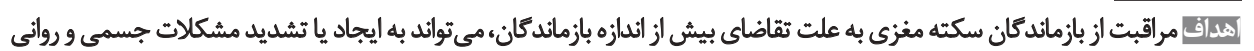

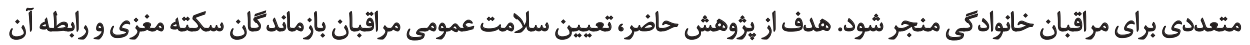
بامشخصات جمعيتشيناختى بوده استيت.

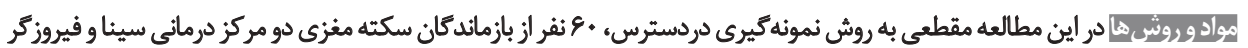

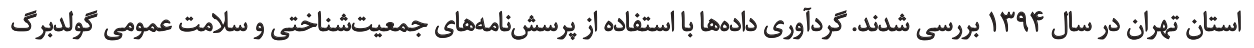

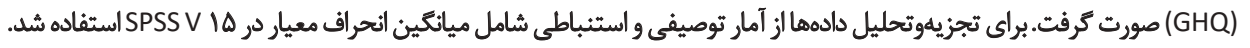

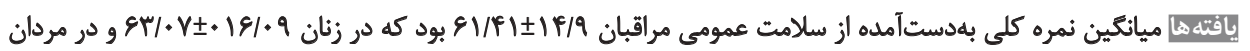

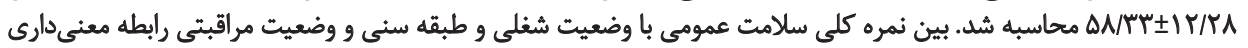

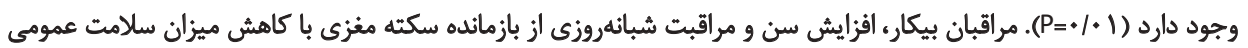

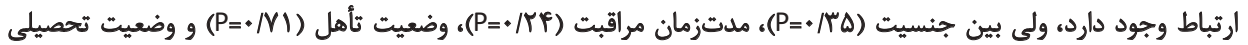

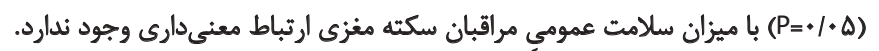

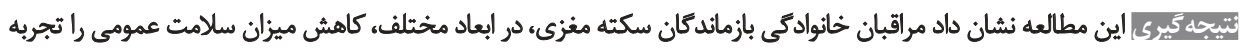

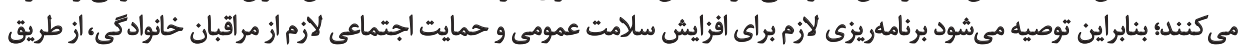
سياست كثناران حوزه سلامت و درمان انجام شُودي.
\end{abstract}

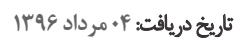

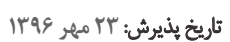

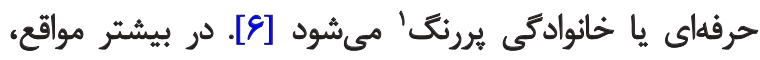

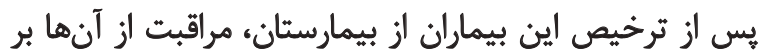

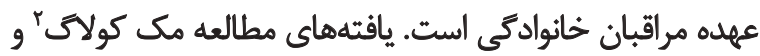

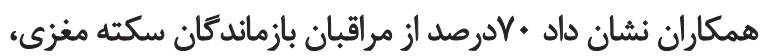

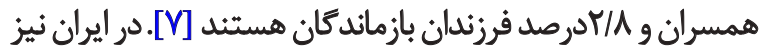

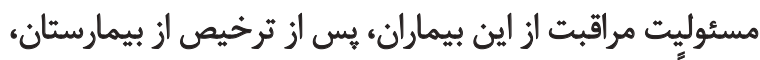

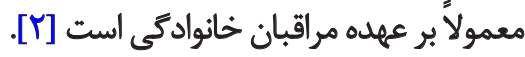

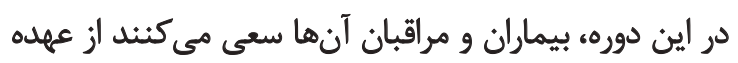

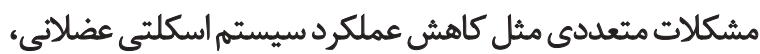

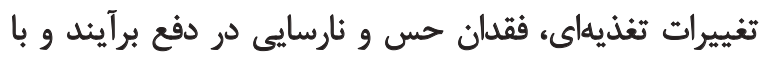

1. Emerge

2. McCullag

مقدمه

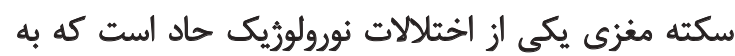

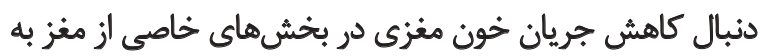

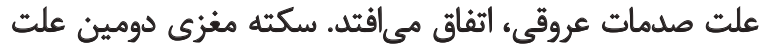

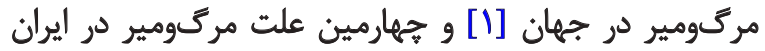

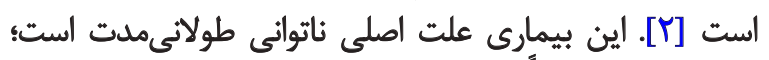

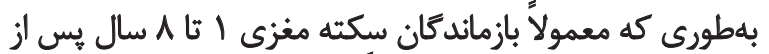

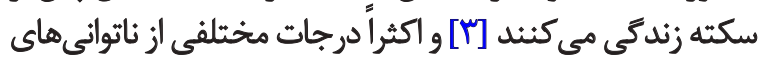

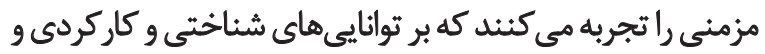

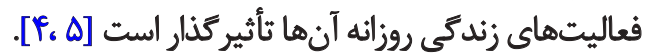
بر اساس ميزان صدمه حاصل الز بيمارى، بيماران مبتلانيازمند

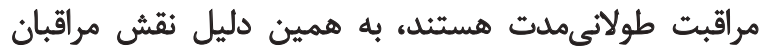

$$
\text { ㄷ..…... }
$$


مراقبان خانوادگى مى شود و نظر به اينكه مسئوليت اصلى درمان و

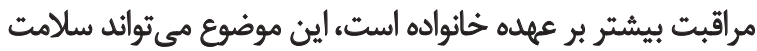

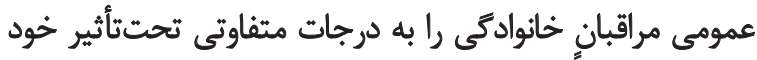

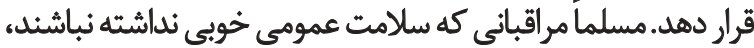

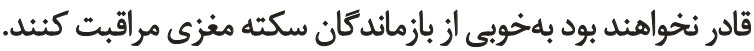

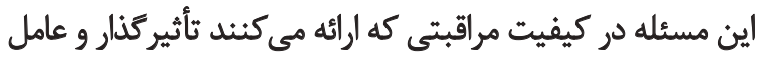

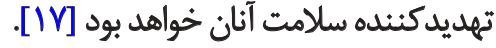

بدون شك، سلامت عمومى مراقبان خانوادكى بازماندكان

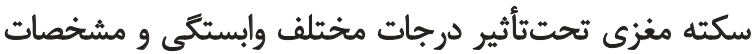

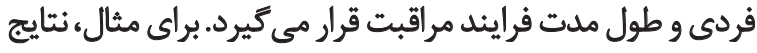

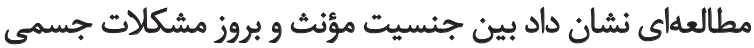

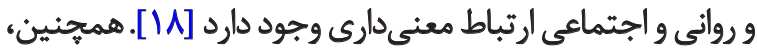

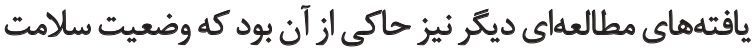

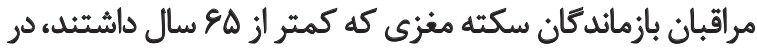

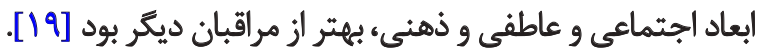

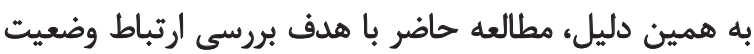

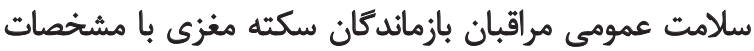
جمعيتشناختى انجام شده است.

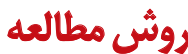

اين مطالعه از نوع توصيفى تحليلي است كه به روش دردسترس،

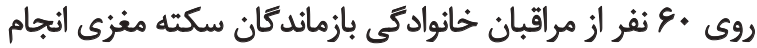

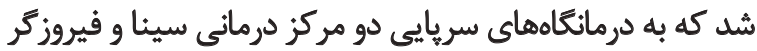

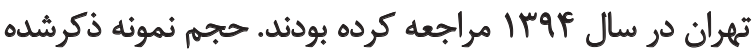

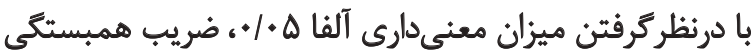

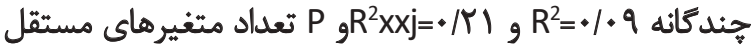

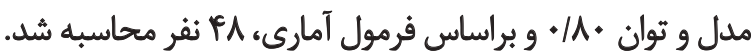

$$
N=\left(\frac{Z(1-\alpha / 2)}{\omega}\right)^{2}\left(\frac{1-R^{2}}{1-R_{x \times j}^{2}}\right)+P+1
$$

با توجه به احتمال ريزش نمونهها، تعداد قطعى حجم نمونه

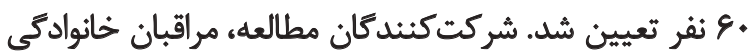

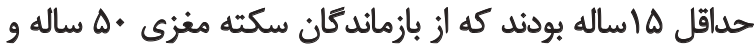

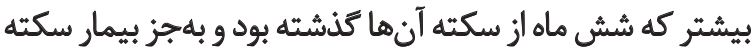

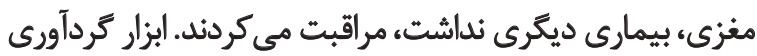

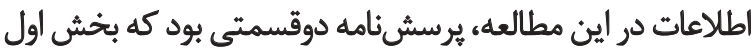

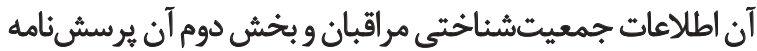

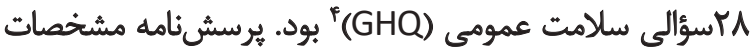

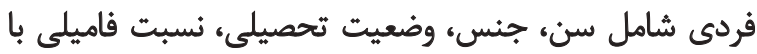

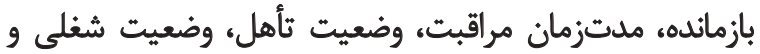

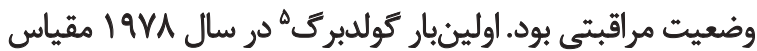

4. General Health Questionnaire(GHQ)

5. Goldberg
تغييرات زندگى روزانه خود سازگًار شوند [1]]؛ به همين دليل

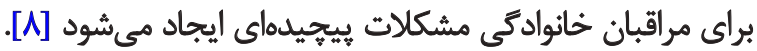

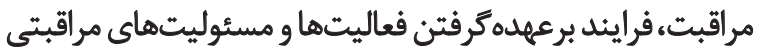

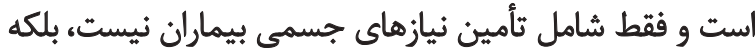

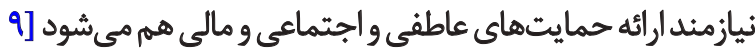

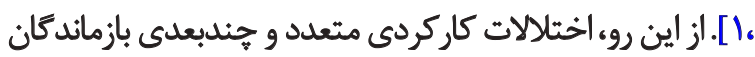

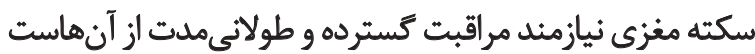

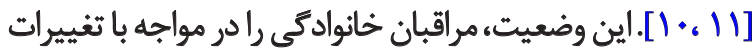

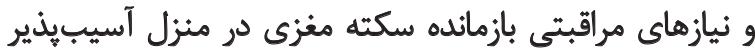

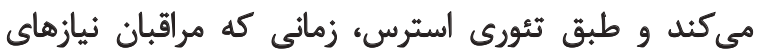

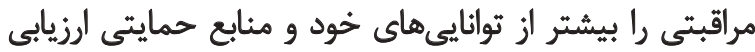

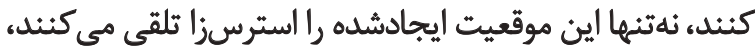

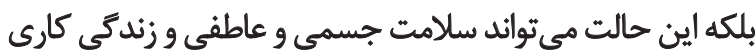

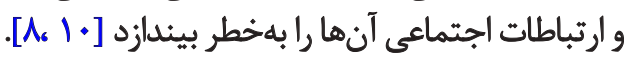

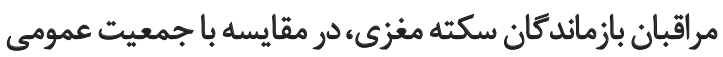

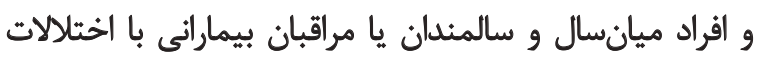

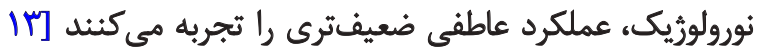

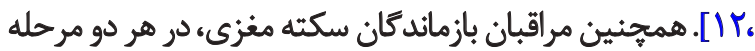

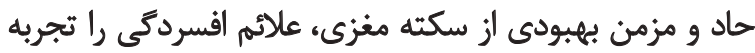

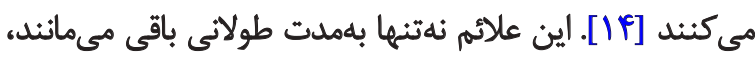

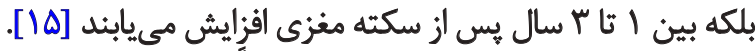

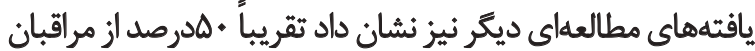

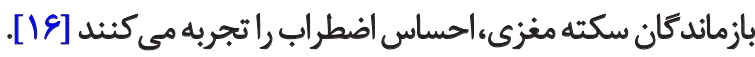
يافتهاي مطالعه مولاكلو" و همكاران نيز مشخص كرد بين

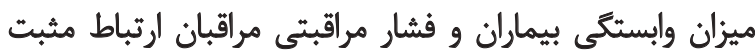

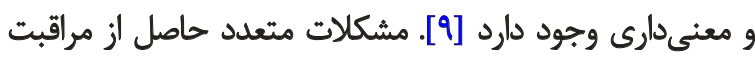

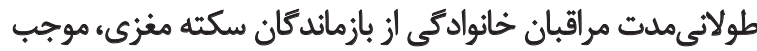

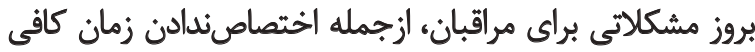

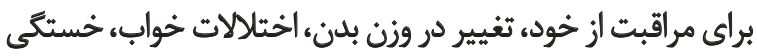

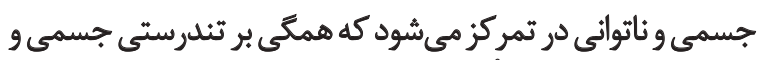

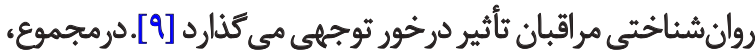

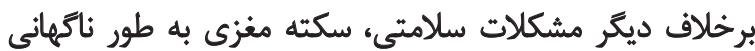

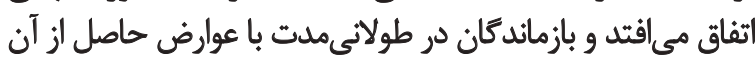

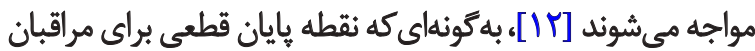

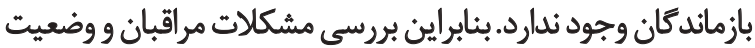

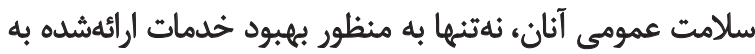

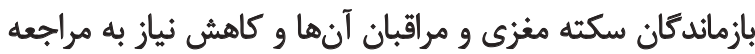

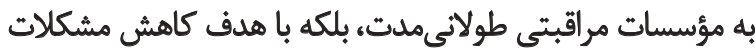

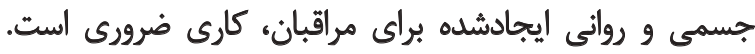

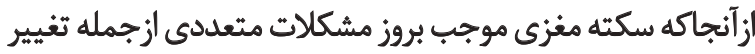

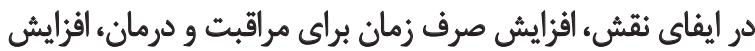

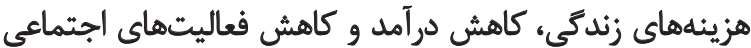

3. Mollaoglu 


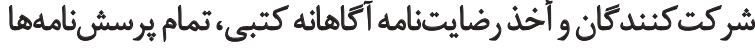

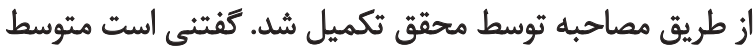

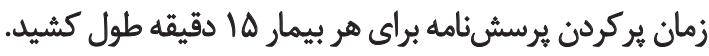
براي تجزيهوتحليل آمارى از نرمافزار SPSS نسخه 10 و و

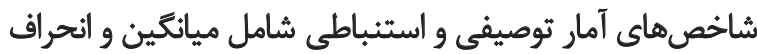

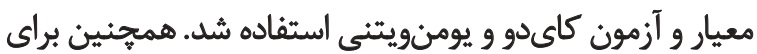

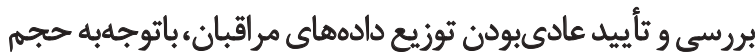

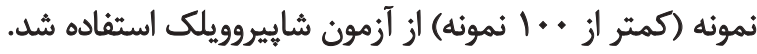

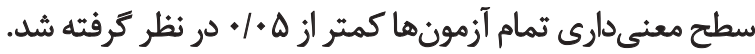

يافتهما

درمجموع •و شركت كننده در اين مطالعه بررسى شدند كه

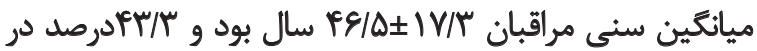

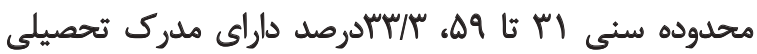

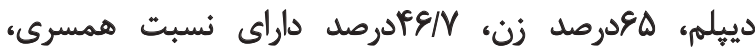
مVI/V

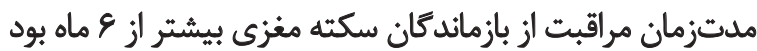

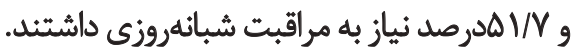

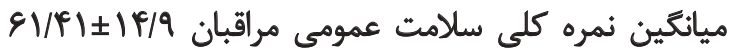

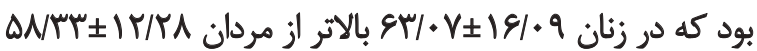
بهدست آمد كه به معناي داشتن مشكان

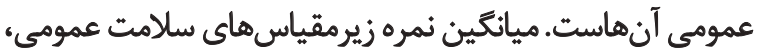
به تفكيك جنسيت در جدول شماره إلرائه شده است. نتيجه آزمون يومنويتنى و كاى بدو نشان داد بهطوركلى بلى بين

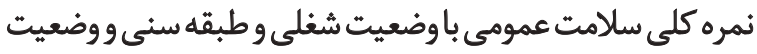

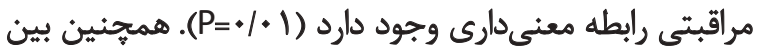

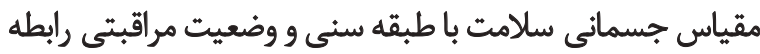

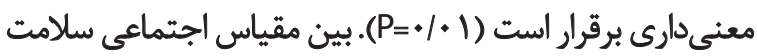

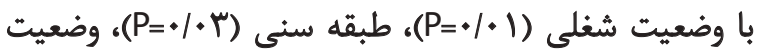

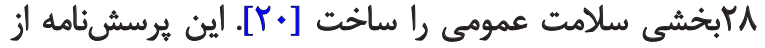

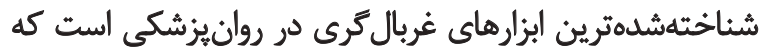

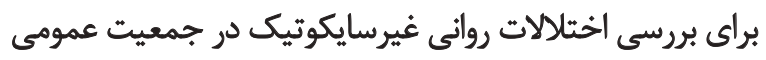

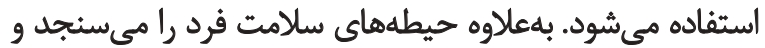

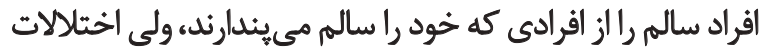

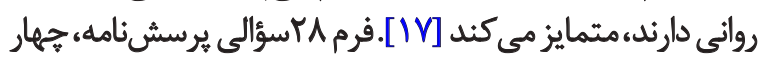

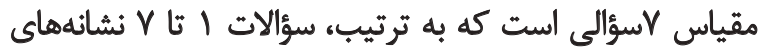

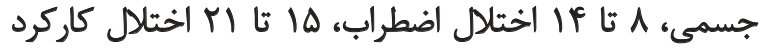

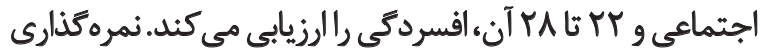

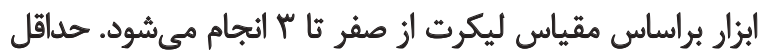

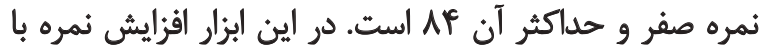

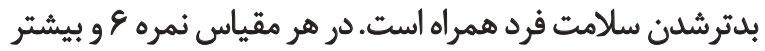

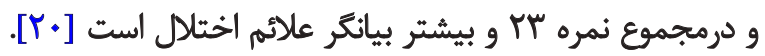

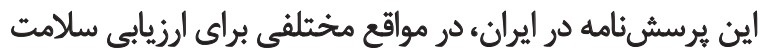

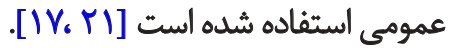
روايى اين يرسشنامه در مطالعات قبلى بررسى و تأييد شده

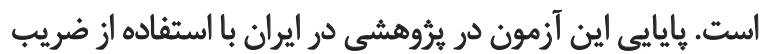

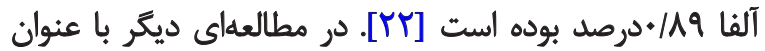
"ابررسى شيوع اختلالات ذهنى در مناطق شهرى نطنز اصفهانه)،

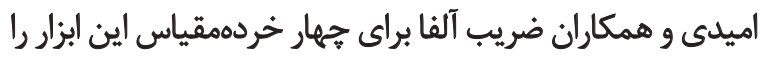

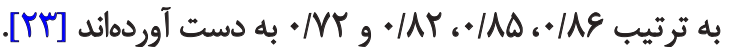
يس از تأييد كميته اخلاق و ارائه معرفىنامه رسمى از معاونت

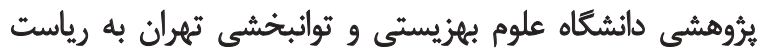

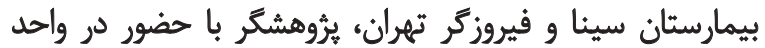

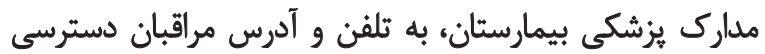

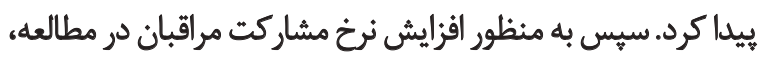

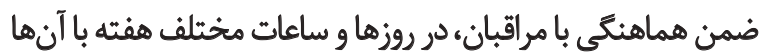

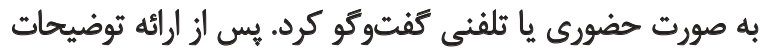

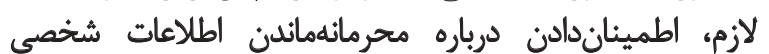

جدول ا. ميانكّين و انحراف معيار سلامت عمومى مراقبان خانوادكى بازماندكان سكته مغزى به تفكيك مرد و زن

\begin{tabular}{|c|c|c|c|}
\hline \multicolumn{3}{|c|}{ توزيع فراوانى } & \multirow{3}{*}{ سلامت عمومى مراقبان } \\
\hline تعداد كل & زن & 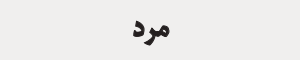 & \\
\hline ميانغين 土|نحراف استاندارد & ميانكين =|أنحراف إيثاندارد & ميانئين ||نحراف استاندارد & \\
\hline $8 / / 41 \pm 16 / 9$ & $94 / . V \pm 19 / .9$ & $\Delta N M T \pm I T / Y A$ & سلامت عمومى (كل) \\
\hline $\mid \& / A \pm F / q$ & $\mid V / r \Delta \pm \Delta / r$ & $1 \Delta / \neg \Delta \pm r / I r$ & خردهمقياس جسمائى \\
\hline $\mid V / \varepsilon \pm F / \varepsilon$ & $I V / q Y \pm F / Y$ & $1 \varepsilon / 9 \pm F / \Delta$ & خردهمقياس اضطراب \\
\hline $\mid f / \lambda \Delta \pm r / V$ & $\| f / M \pm r / \varphi$ & $\mid r / \cdot \Delta \pm r / N$ & خردهمقياس اجتماعى \\
\hline $1 r / Y " \pm f / 9$ & $1 W / \Delta \pm \pm 1 \% / \Delta$ & $\mid T / V E \pm T / A F$ & خردمثقياس افسردمى \\
\hline
\end{tabular}

L الم 
جدول r. بررسى رابطه ميانكين رتبه سلامت عمومى و زيرمقياسهاى آن با مشخصات جامعهشئاختى مراقبان خانوادتى بازمائدكان سكته مغزى

\begin{tabular}{|c|c|c|c|c|c|c|c|c|c|c|c|}
\hline آآزهون & افسردكّى & أزمون & اجتماعى & أمارى آزمون & اضطراب & آزارمون & جسماتى & آزأمون & سلامث & \multicolumn{2}{|l|}{ متغير } \\
\hline \multirow[t]{2}{*}{$\cdot / \mathbb{A}^{*}$} & rap & $+1+A^{*}$ & ro/rq &.$/ M E *$ & TV/V & $.18 \Delta^{\circ}$ & $r q /$ &.$/ N \omega^{*}$ & rVPeV & مرد & S. \\
\hline & $M V / q$ & & 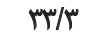 & & $m / 9$ & & $r \mid r$ & & $r V / r$ & ij & \\
\hline \multirow[b]{2}{*}{.$/ M *$} & $M \varphi /+1$ & & TNF & & $r+/ F$ & & $r+/ r$ & & Tr/gV & ع ماه & \\
\hline & $r v / r$ & $+/ V \Delta^{*}$ & $r+/ V$ & $+/ 1$ & $\mathrm{r} / \mathrm{s}$ & $+/ 11$ & MV/ & $\cdot / M$ & $M / N$ & بيش ازعماه & है. \\
\hline \multirow{3}{*}{.$/ \mathrm{u}^{*}$} & $m+/ F$ & & $r V / r$ & & $r / T$ & & $r q / r$ & & หม/ & مجرد & ?̊ \\
\hline & & $\cdot / r \Delta^{*}$ & & - $18 *$ & & $\cdot M^{*}$ & & $\cdot M$ & & & $83^{3}$ \\
\hline & $r . / \Delta$ & & 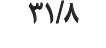 & & $r 9 / \wedge$ & & $r * / q$ & & MVlor & هثtهل & $\frac{3}{3}$ \\
\hline \multirow{3}{*}{$+/ f^{*}$} & WV/r & & $r+r$ & & $m / l$ & & $m / q$ & & $r / / r$ & شاغل & is \\
\hline & & $.1 \cdot 1 *$ & & $.1 .0^{\circ}$ & & $.1 .9^{\circ}$ & & $+1 \cdot 1^{*}$ & & & 4 \\
\hline & $r 1 / \Delta$ & & $m / 9$ & & $M T / 9$ & & MT/8 & & $M / \Delta$ & ييكار & -3 \\
\hline \multirow{3}{*}{$.1 .90 *$} & $r v / q$ & & $T V / T^{e}$ & & $r \cdot / e$ & & $M V / \Delta$ & & rq/ & M-Q9 & 코ํ. \\
\hline & & $\cdot 1 \cdot 1^{* * *}$ & & $.1 .9 * *$ & & $.1 .1^{* *}$ & & $.1 .1^{* *}$ & & & 3 \\
\hline & $r \otimes / \%$ & & $\mathrm{rr} / \mathrm{S}$ & & re/ & & พq/ब & & Pile & بيش از•• & $\stackrel{3}{3}$ \\
\hline \multirow{5}{*}{$\cdot / \Delta V^{* *}$} & re/ & & $P H / V$ & & $r N r$ & & $r q / T$ & & $P T / T$ & بيسواد & \\
\hline & $m / / r$ & & $r \mid r$ & & $m / \cdot r$ & & $r+r$ & & $m / q$ & ابتدايع & ई̊ \\
\hline & ra/s &.$/ . r *$ & $r \Delta / r$ & $\cdot / \Gamma \Lambda^{* * *}$ & $r V / V$ & . $118 * *$ & $M T / *$ & $.1 \cdot \theta^{* *}$ & TNV & دييلم & 8 \\
\hline & $r E / V$ & & $r \Delta / V$ & & $r \Delta / r$ & & $r M / f$ & & $r M / r$ & كارشناسى & $\frac{3}{3}$ \\
\hline & $1 Q / \Delta$ & & rel.. & & $r q /$. & & $\mathrm{rV} /$. & & $\mathrm{rI} /$. & كارشناسيى & \\
\hline \multirow{5}{*}{$\cdot \mathbb{A}^{*}$} & rq/8 & & $r \Delta / \lambda$ & & $\mathrm{rI/S}$ & & $\mathrm{Tr} / \mathrm{I}$ & & $M T / T$ & همسر & \\
\hline & $r q / r$ & & $r=/ 0$ & & $M / s$ & & $r q / V$ & & $r \cdot 11$ & دختر & 3. \\
\hline & $r \cdot / M$ & $.1 \cdot v^{*}$ & $19 / r$ &.$N \Delta^{*}$ & rels & $.10 \%$ & $r e / q$ &.$/ m v^{*}$ & $\pi / \pi$ & يسر & -3 \\
\hline & PV/Q & & $P r / r$ & & $P \cdot / V$ & & $\Delta . / T$ & & $p q$. & مادر & $3^{3 i}$ \\
\hline & $\pi \%$ & & $r \% /$. & & $18 \%$ & & $r . \%$ & & rel. & يلر & \\
\hline \multirow{3}{*}{$.1 .1^{*}$} & 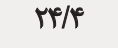 & & $M / r$ & & $r E / T$ & & $r E$ & & $r e / r$ & روزائه & \\
\hline & $10 / 9$ & $.1 .1^{*}$ & $r r / q$ & $+1+0^{*}$ & $M T / \cdot A$ & $.1 .1^{\circ}$ & $r+18$ & $+1 \cdot 1^{*}$ & $18 / 9$ & شبائه & $\begin{array}{l}3 \\
3 \\
2 \\
2\end{array}$ \\
\hline & rq/p & & $r V / r$ & & role & & rele & & พथ/ & شبائهروزى & $y$ \\
\hline
\end{tabular}


مراقبتى، بار سنكَين مسئوليتيذيرى، نكرانى دائمى و اين

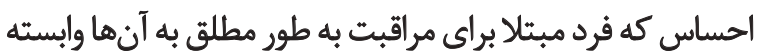

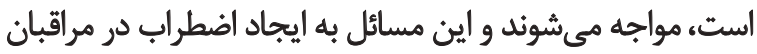

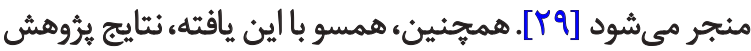

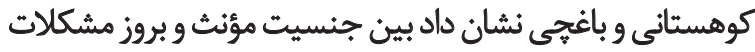

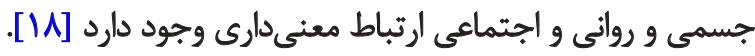

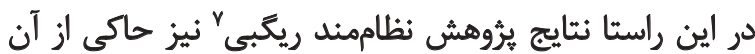

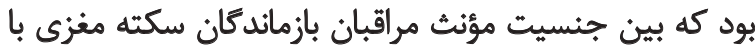

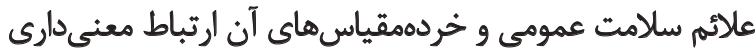

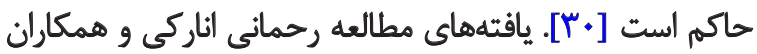

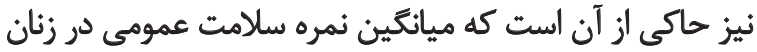

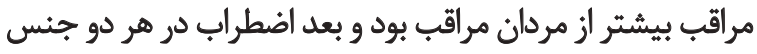

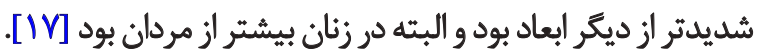

ازجمله دلايل همسويى يافتههاى مطالعه حاضر با مطالعات

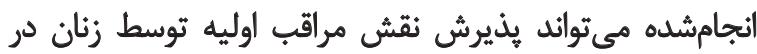

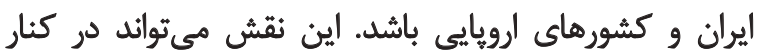

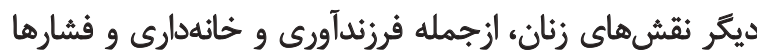

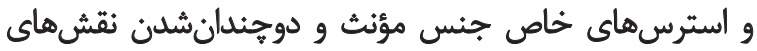

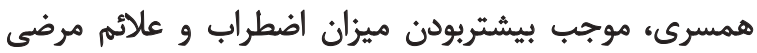

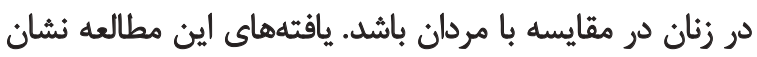

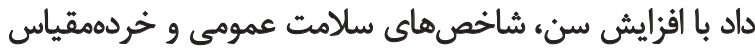

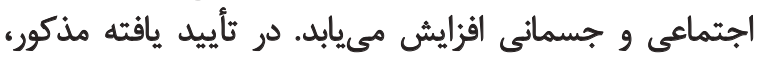

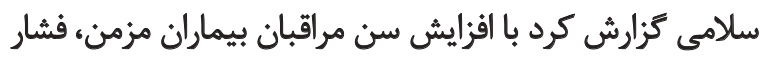

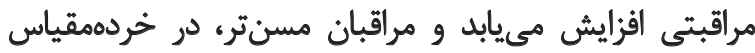

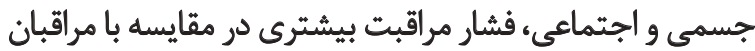

جوانتر تجربه مى اجنئد [اسب].

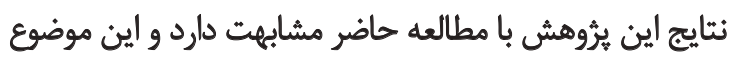

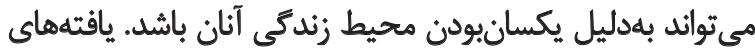

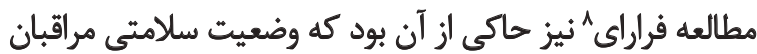

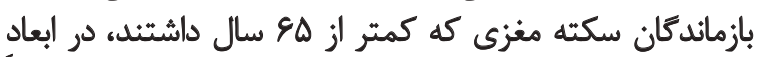

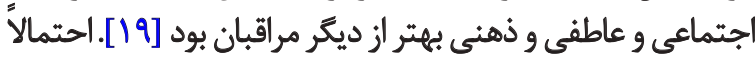

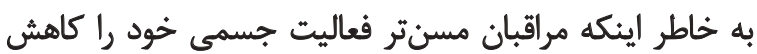

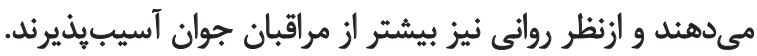

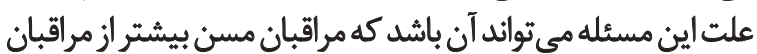

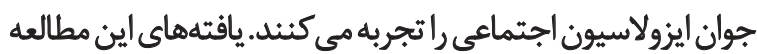

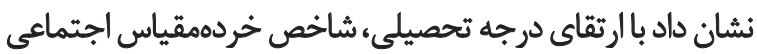

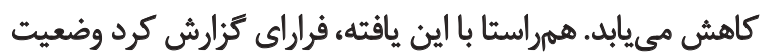

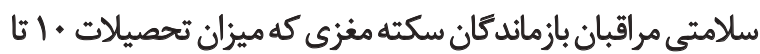

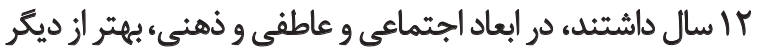

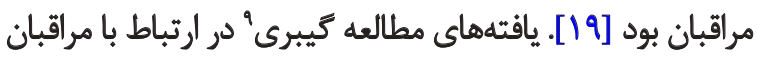

\section{Rigby}

8. Ferreira

9. Gbiri

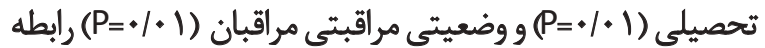

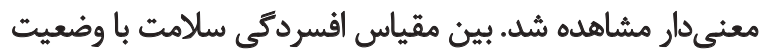

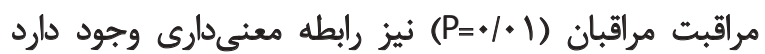

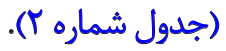

$\stackrel{\leftrightarrow}{4}$

مطالعه حاضر با هدف بررسى رابطه سلامت عمومى در مراقبان

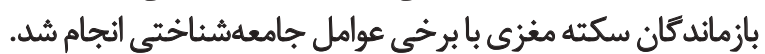

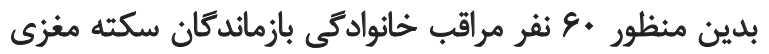

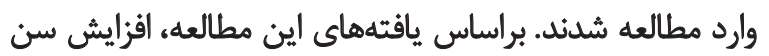

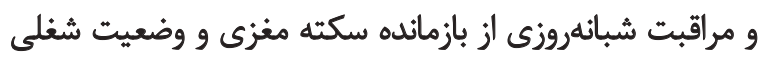

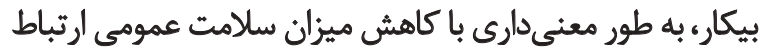

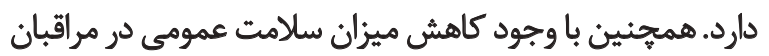

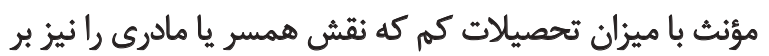
عهده داشتند، اين ارتباط به لحاظ آمارى معنى دار نبود.

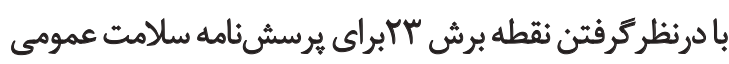

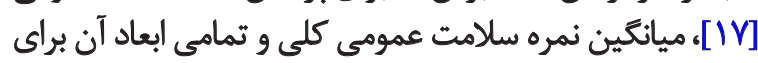

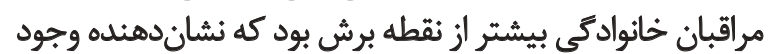

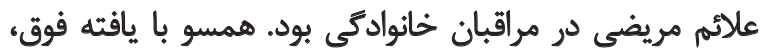

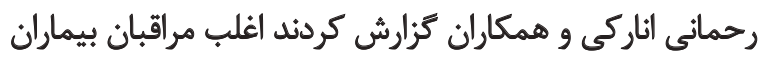

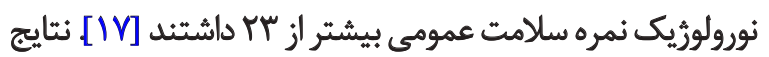

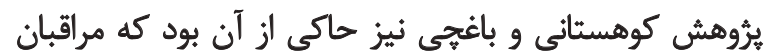

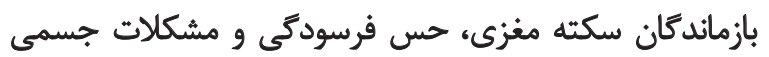

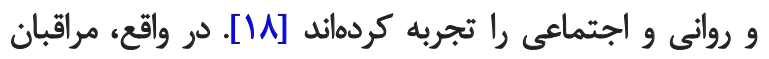

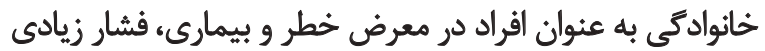

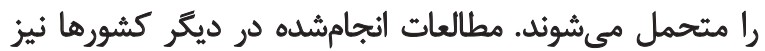

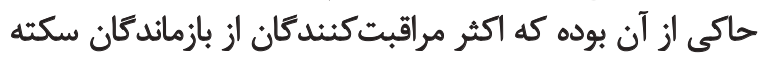

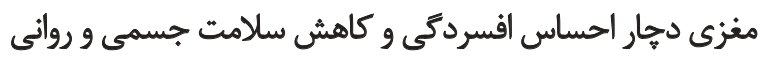

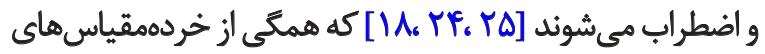

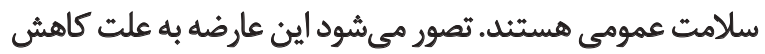

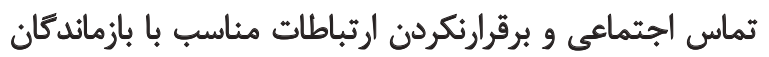

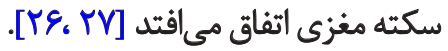
ازجمله دلايل همسويى يافتههاى مطالعه حاضر با مطالعات

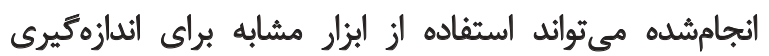

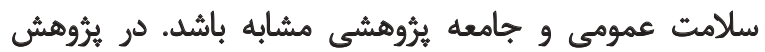

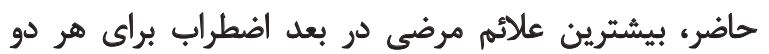

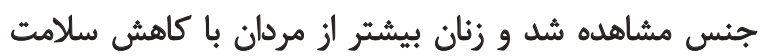

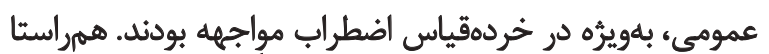

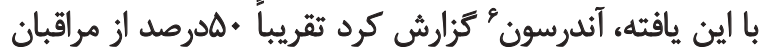

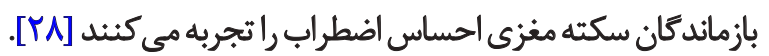

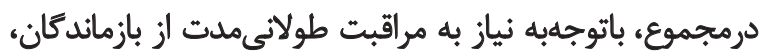

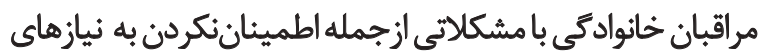

6. Anderson 
درزمينه مراقبت خانوادگى گروهى از طريق يرستاران و يزّشكان

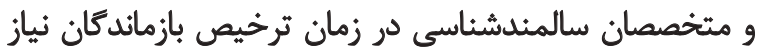

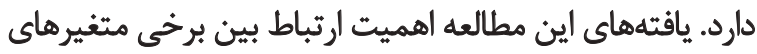

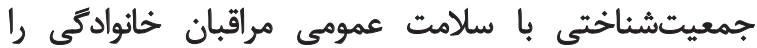

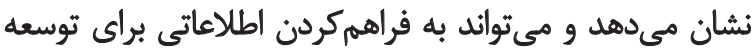

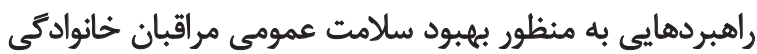

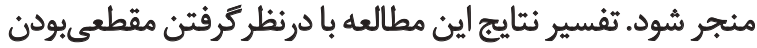

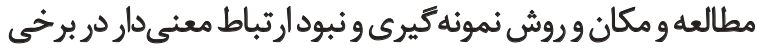

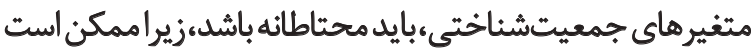

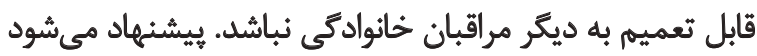

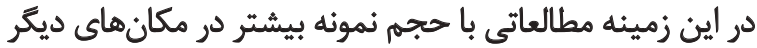

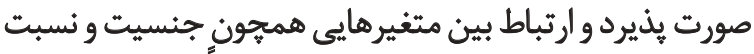
فاميلى با بيمار با ميزان سلامت عمومى مجداً بردئ بررسى شود.

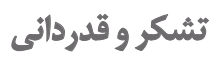

اين مقاله، حاصل طرح تحقيقائى إياننامه كارشناسى ارشد

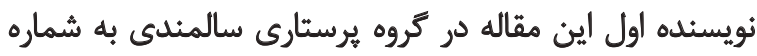

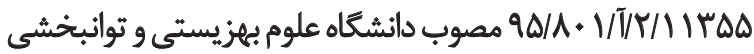

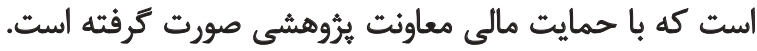

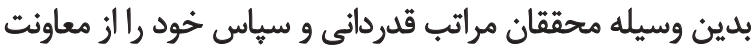

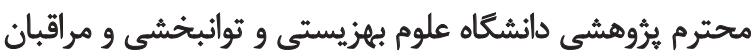

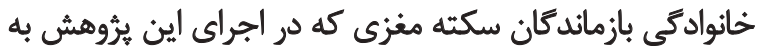

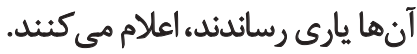

خانكى مراقبت كنيده از بازماندكان سكته مغزى نيز همسو با يافته

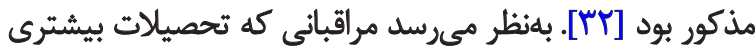

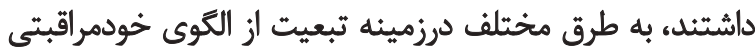

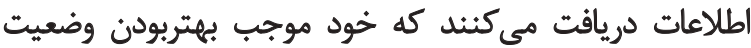

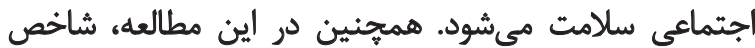

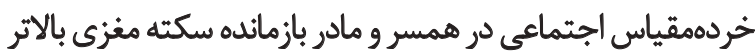

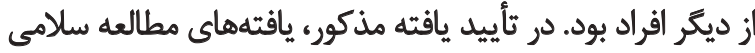

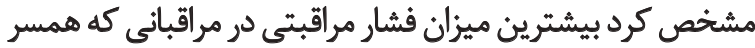

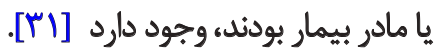

به نظر مىرسد همسران يا مادران مراقب به علث نداشتن وقت

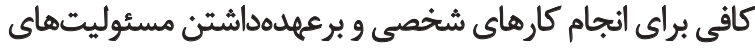

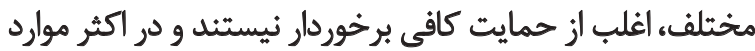

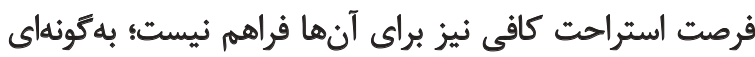

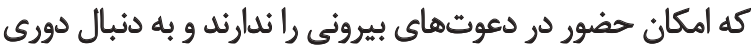

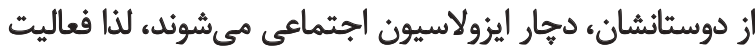

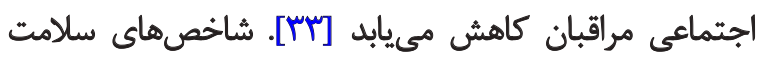

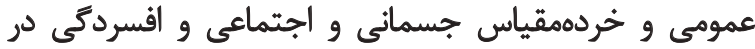

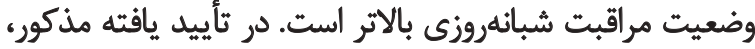

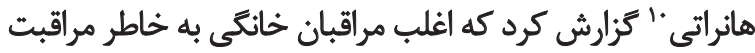

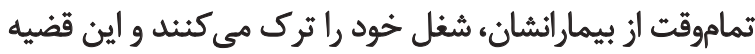

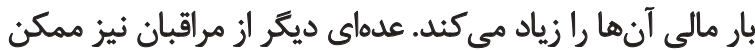

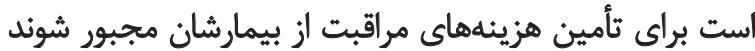

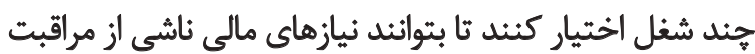
شبانهروزى از بيمارشان را برآورده كنيند. به دنبال مسائل ينائل يادشدها.

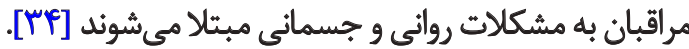

\section{نتيجه كيرى نمهايي}

يافتههاى اين مطالعه نشان داد افرادى كه از بازماندكان

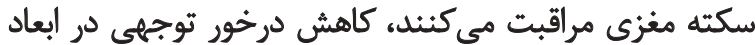

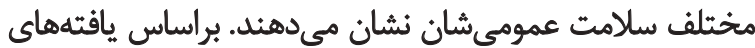

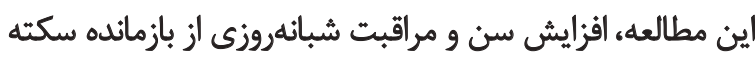

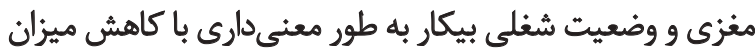

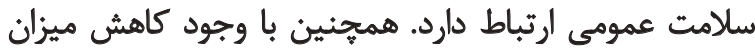

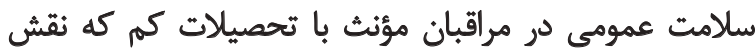

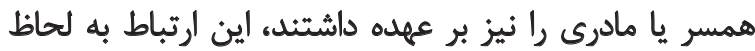

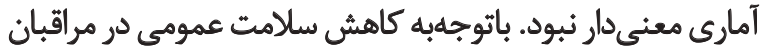

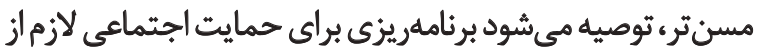

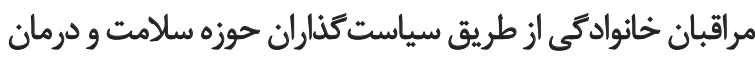

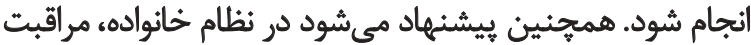

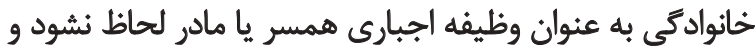

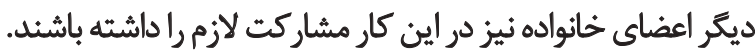
اين موضوع به فراهم كردن آموزشهاى مربوط و فرهنكسازى 


\section{References}

[1] Gorgulu U, Polat U, Kahraman BB, Ozen S, Arslan E. Factors aff fecting the burden on caregivers of stroke survivors in Turkey. Medical Science and Discovery. 2016; 3(4):159-65. doi: 10.17546/ msd.18253

[2] Azam Zeinali AD. [Estimation relationship uncertainty with coping strategy and functional independence in patients stroke who were reffered to Sina and Firouzgar hospitals (Persian)] [MSc. thesis]. Tehran: University of Social Welfare and Rehabilitation Sciences; 2016.

[3] Costa TFd, Gomes TM, Viana LRdC, Martins KP, Costa KNdFM. [Stroke: patient characteristics and quality of life of caregivers (Portuguese)]. Revista Brasileira de Enfermagem. 2016; 69(5):9339. doi: 10.1590/0034-7167-2015-0064.

[4] Hesamzadeh A, Dalvandi A, Maddah SB, Khoshknab MF, Ahmadi F. Family adaptation to stroke: a metasynthesis of qualitative research based on double ABCX model. Asian Nursing Research. 2015; 9(3):177-84. doi: 10.1016/j.anr.2015.03.005

[5] Dalvandi A, Heikkilä K, Maddah S, Khankeh H, Ekman SL. Life experiences after stroke among Iranian stroke survivors. International Nursing Review. 2010; 57(2):247-53. doi: 10.1111/j.14667657.2009.00786.x

[6] Araújo JS, Da SILVA SED, De Santana ME, Dos Santos LS, Sousa $\mathrm{RF}$, Vasconcelos EV, et al. [The parallel side of caring revealed by the representations of caregivers of people suffering from stroke (Portuguese)]. Revista Brasileira de Ciências da Saúde. 2015; 18(2):109-14. doi: 10.4034/rbcs.2014.18.02.03

[7] McCullagh E, Brigstocke G, Donaldson N, Kalra L. Determinants of caregiving burden and quality of life in caregivers of stroke patients. Stroke. 2005; 36(10):2181-6. doi: 10.1161/01. str.0000181755.23914.53

[8] Rahmani AH, Mahmoudi GR, Roohi G, Asayesh H, Nasiri H, Rakhshani H. [General health status of neurologic patients'caregivers and the related factors (Persian)]. Journal of Research Development in Nursing And Midwifery. 2013; 9(2):4955 .

[9] Mollaoğlu M, Özkan Tuncay F, Kars Fertelli T. The Burden of caregivers of stroke patients and the factors affecting the burden. Archives of Neuropsychiatry. 2011; 49(1):169-74.

[10] Opara JA, Jaracz K. Quality of life of post-stroke patients and their caregivers. Journal of Medicine and Life. 2010; 3(3):216-20. PMCID: PMC3018998

[11] Tuna HM, Olgun N. The Role of perceived social support in burnout in patients with stroke care. Journal of Hacettepe University Nursing of Faculty. 2010;17(1):041-52.

[12] Simon C, Kumar S, Kendrick T. Cohort study of informal carers of first-time stroke survivors: Profile of health and social changes in the first year of caregiving. Social science \& medicine. 2009; 69(3):404-10. doi: 10.1016/j.socscimed.2009.04.007

[13] Chow SK, Wong FK, Poon CY. Coping and caring: support for family caregivers of stroke survivors. Journal of Clinical Nursing. 2007; 16(7b):133-43. doi: 10.1111/j.1365-2702.2006.01711.x

[14] Han B, Haley WE. Family caregiving for patients with stroke review and analysis. Stroke. 1999; 30(7):1478-85. doi: 10.1161/01. str.30.7.1478
[15] Visser-Meily JA, Rhebergen ML, Rinkel GJ, van Zandvoort MJ, Post MW. Long-term health-related quality of life after aneurysmal subarachnoid hemorrhage relationship with psychological symptoms and personality characteristics. Stroke. 2009; 40(4):1526-9. doi: 10.1161/strokeaha.108.531277

[16] Anderson JA, Willson P. Clinical decision support systems in nursing: Synthesis of the science for evidence-based practice. Computers Informatics Nursing. 2008; 26(3):151-8. doi: 10.1097/01.ncn.0000304783.72811.8e

[17] Rahmani Anaraki H, Mahmoodi G R, Rouhi G, Asayesh H, Nasiri H, Rakhshani H. [General health status of neurologic patients' caregivers and the related factors (Persian)]. Journal of Research Development in Nursing and Midwifery. 2013;9(2):49-55.

[18] Koohestani H, Baghcheghi N. [Burn out in caregivers of patients with stroke and its related factors (Persian)]. Hakim Health System Research Journal. 2012;14(4):242-8.

[19] Ferreira CG, Alexandre TdS, Lemos ND. [Factors associated with the quality of life of caregivers of elderly individuals in home care (Portuguese)]. Saúde e Sociedade. 2011; 20(2):398-409. doi: 10.1590/S0104-12902011000200012

[20] Goldberg DP, Hillier VF. A scaled version of the General Health Questionnaire. Psychological Medicine. 1979; 9(01):139-45. doi: 1017/s0033291700021644

[21] Navidian A, Masoudi G, Mousavi S. [Work-related stress and the general health of nursing staffs in zahedans' hospitals emergency wards (Persian)]. Journal of Kermanshah University of Medical Sciences. 2005; 9(3):22-8.

[22] Kaviani H, Mohit A. [Interviews and psychological tests (Persian)]. Tehran: Sana Publisher; 2002.

[23] Omidi A, Sazvar SA, Akashe G. [Epidemiology of Mental Disorders in Urbanized Areas of Natanz (Persian)]. Iraninan Psychiatry and Clinical Psychology. 2004; 8(4):32-8.

[24] Baumann M, Lurbe-Puerto K, Alzahouri K, Aïach P. Increased residual disability among poststroke survivors and the repercussions for the lives of informal caregivers. Topics in Stroke Rehabilitation. 2011; 18(2):162-71. doi: 10.1310/tsr1802-162

[25] Salter K, Zettler L, Foley N, Teasell R. Impact of caring for individuals with stroke on perceived physical health of informal caregivers. Disability and Rehabilitation. 2010; 32(4):273-81. doi: 10.3109/09638280903114394

[26] Parag V, Hackett ML, Yapa CM, Kerse N, McNaughton H, Feigin VL, et al. The impact of stroke on unpaid caregivers: Results from The Auckland Regional Community Stroke study, 2002-2003. Cerebrovascular Diseases. 2008; 25(6):548-54. doi: $10.1159 / 000131673$

[27] Jönsson A-C, Lindgren I, Hallström B, Norrving B, Lindgren A. Determinants of quality of life in stroke survivors and their informal caregivers. Stroke. 2005; 36(4):803-8. doi: 10.1161/01. str.0000160873.32791.20

[28] Anderson CS, Linto J, Stewart-Wynne EG. A population-based assessment of the impact and burden of caregiving for long-term stroke survivors. Stroke. 1995;26(5):843-9.

[29] Kausar R. Cognitive appraisal, coping and psychological distress in carers of physically disabled people in Pakistan. Journal of Behavioural Sciences. 2001;12(1-2):47-68. 
[30] Rigby H, Gubitz G, Phillips S. A systematic review of caregiver burden following stroke. International Journal of Stroke. 2009;4(4):285-92. doi: 10.1111/j.1747-4949.2009.00289.x

[31] Salmani A, Ashketorab T, Hasanvand SH. [The Burden of Caregiverand Related Factorsof Oncology Patients of Shah Vali Hospital (Persian)]. Advances in Nursing \& Midwifery. 2015; 24(2):11-7.

[32] Gbiri CA, Olawale OA, Isaac SO. Stroke management: Informal caregivers' burdens and strians of caring for stroke survivors. Annals of Physical and Rehabilitation Medicine. 2015; 58(2):98-103. doi: 10.1016/j.rehab.2014.09.017

[33] Scannell AU. The longterm psychosocial impacts of caregiving on the caregivers of persons with stroke [PhD thesis]. Portland: Portland State University; 1989

[34] Hanratty B, Holland P, Jacoby A, Whitehead M. Review article: financial stress and strain associated with terminal cancer -a review of the evidence. Palliative Medicine. 2007; 21(7):595-607. doi: 10.1177/0269216307082476 
